\title{
Immunogenicity of heterologous prime/booster- inactivated and adenoviral-vectored COVID-19 vaccine: real-world data
}

\section{Nasamon Wanlapakorn}

Center of Excellence in Clinical Virology, Faculty of Medicine, Chulaongkorn University Nungruthai Suntronwong

Center of Excellence in Clinical Virology, Faculty of Medicine, Chulaongkorn University Harit Phowatthanasathian

Center of Excellence in Clinical Virology, Faculty of Medicine, Chulaongkorn University

Ritthideach Yorsang

Center of Excellence in Clinical Virology, Faculty of Medicine, Chulaongkorn University https://orcid.org/0000-0001-9391-663X

\section{Thanunrat Thongmee}

Center of Excellence in Clinical Virology, Faculty of Medicine, Chulaongkorn University

\section{Preeyaporn Vichaiwattana}

Center of Excellence in Clinical Virology, Faculty of Medicine, Chulaongkorn University Chompoonut Auphimai

Center of Excellence in Clinical Virology, Faculty of Medicine, Chulaongkorn University Lakkhana Wongsrisang

Center of Excellence in Clinical Virology, Faculty of Medicine, Chulaongkorn University Sirapa Klinfueng

Center of Excellence in Clinical Virology, Faculty of Medicine, Chulaongkorn University Natthinee Sudhinaraset

Center of Excellence in Clinical Virology, Faculty of Medicine, Chulaongkorn University

Yong Poovorawan ( $\sim$ Yong.P@chula.ac.th )

Center of Excellence in Clinical Virology, Faculty of Medicine, Chulaongkorn University, FRS(T), the Royal Society of Thailand, Sanam Sueapa, Dusit

\section{Article}

Keywords: SARS-CoV-2, heterologous vaccine regimens, spike RBD-specific IgG

Posted Date: September 10th, 2021

DOI: https://doi.org/10.21203/rs.3.rs-785693/v2 
License: (c) (i) This work is licensed under a Creative Commons Attribution 4.0 International License. Read Full License 


\section{Abstract}

Limited data are available on the responses to heterologous vaccine regimens for SARS-CoV-2, especially among countries using inactivated and adenoviral-vectored vaccines. A total of 77 participants who received heterologous prime/booster-inactivated COVID-19 vaccine (CoronaVac) and adenoviral-vectored vaccine (AZD1222) were enrolled in our study. There were two comparison groups vaccinated with the homologous CoronaVac $(N=80)$ and AZD1222 $(N=80)$ regimen. All sera samples were tested for SARSCoV-2 spike receptor-binding-domain (RBD) IgG using a chemiluminescent microparticle immunoassay (CMIA). The neutralizing activity in a subset of serum samples was tested against the original Wuhan strain and variants of concern, B.1.1.7, B.1.617.2 and B.1.351, using an enzyme-linked immunosorbent assay (ELISA)-based surrogate virus neutralization test (sVNT). The heterologous CoronaVac/AZD1222 vaccine induced higher levels of spike RBD-specific lgG than that of two-dose homologous CoronaVac or AZD1222 vaccines $(p<0.001)$. Sera samples of the CoronaVac/AZD1222 vaccine recipients elicited higher neutralizing antibody activity against the original Wuhan and all variants of concern than in the recipients of the two-dose CoronaVac. Nevertheless, there were no difference in neutralizing activity against the original Wuhan and B.1.1.7 strain among heterologous CoronaVac/AZD1222 and homologous AZD1222 vaccine recipients.

\section{Introduction}

Limited data are available on the responses to heterologous vaccine regimens for SARS-CoV-2, especially among countries using inactivated and adenoviral-vectored vaccines. In the Com-COV trial, heterologous prime-booster combinations of the adenoviral-vectored (AZD1222) and mRNA vaccines induced higher serum anti-spike receptor-binding domain (RBD) and neutralizing antibody titers than the homologous efficacy-proven adenoviral-vectored two-dose regimens [1]. The CombiVacS trial found that the Pfizer's BNT162b2 mRNA vaccine given as a second dose in individuals prime vaccinated with AZD1222 induced a robust immune response, with an acceptable and manageable reactogenicity profile [2].

Some countries have recommended the heterologous vaccine regimen, offering the mRNA vaccines as a second dose to young people who have already received the AZD1222 vaccine because of concerns about vaccine-induced thrombotic thrombocytopenia (VITT) [3]. The preliminary results showed that the heterologous vaccine regimen (AZD1222/BNT162b2) induced higher humoral and cellular responses to SARS-CoV-2 compared to the homologous vaccine regimen (AZD1222/AZD1222) [4]. Further research is required to comprehensively elucidate the immunological implications following different vaccine types and administration schedules.

Thailand has imported CoronaVac, which was developed by Sinovac Life Sciences, Beijing, China, since February 2021. Several phase 3 studies have shown acceptable safety and efficacy against symptomatic COVID-19 following two-dose CoronaVac vaccination [5-7]. Health professionals were the first prioritized group receiving two-dose CoronaVac in Thailand. Adenoviral-vectored vaccine, imported from Korea in March 2021 and later produced from the Siam Bioscience company (Nonthaburi, Thailand) 
was initially prioritized for the elderly above 60 years of age. Preliminary studies have found the two-dose CoronaVac regimen induced a lower, but acceptable, immune response compared to the two-dose AZD1222 regimen; however, there was a significantly shorter waiting period between CoronaVac doses. Thailand started vaccinations with the inactivated CoronaVac on 28 February, 2021, and AZD1222 vaccine on 16 March, 2021, with a 3- to 4-week and 10-week waiting period between doses, respectively. A 10-week waiting period was established based on recommendations of the Thailand FDA and efficacy studies identifying that a waiting period of $<6$ weeks resulted in lower immune stimulation than a period of 10 weeks [8]. The CoronaVac vaccine was associated with a rare focal neurological syndrome characterized by numbness, or sometimes weakness, in the limbs [9]. Although this self-limited adverse event is rare, individuals experiencing this side effect sought another regimen for their second shot, which was AZD1222.

It is possible to mix and match vaccines in specific situations such as a vaccine shortage or for adverse reactions following vaccine administration. This study aims to assess the immunogenicity of heterologous prime/booster inactivated COVID-19 vaccine and the adenoviral-vectored vaccine currently available in Thailand to provide preliminary data on their immunogenicity.

\section{Methods}

\subsection{Study design}

We performed a cross-sectional study in which leftover sera samples from participants seeking antibody testing following vaccination at the Center of Excellence in Clinical Virology, Department of Pediatrics, Faculty of Medicine, Chulalongkorn University between April and July 2021 were further analyzed. Only samples from participants who received heterologous prime/booster inactivated COVID-19 vaccine and adenoviral-vectored vaccine were used. The study protocol was approved by the Research Ethics Committee of the Faculty of Medicine, Chulalongkorn University (IRB number 491/64) and this trial is registered with Thai Clinical Trials Registry (TCTR20210628005). The committee waived the requirement for consent because the samples used were de-identified and anonymous.

We also included two comparison groups vaccinated with the homologous CoronaVac and AZD1222 regimen (IRB no. 192/64, TCTR20210319003) in the analysis. Participants in the comparison groups received CoronaVac or AZD1222 vaccines at the Banphaeo General Hospital, Samutsakorn Province, Thailand, between March and June 2021. Informed consent was obtained during the second-dose vaccination visit. Participants who consented to blood sampling at 21-35 days after full vaccination were scheduled for an extra blood sampling visit at the Banphaeo General Hospital, Samutsakorn Province.

\subsection{Study vaccine}


CoronaVac is an inactivated virus vaccine created from African green monkey kidney cells (Vero cells) that have been inoculated with SARS-CoV-2 (CZ02 strain). The virus was inactivated with $\beta$-propiolactone and finally absorbed onto aluminum hydroxide. Each vial contains $0.5 \mathrm{~mL}$ with $600 \mathrm{SU}$ (equal to $3 \mu \mathrm{g}$ ) of inactivated SARS-CoV-2 virus as antigen [10]. Chimpanzee adenovirus Oxford 1 (ChAdOx1)-vectored vaccine (AZD1222) from Oxford/AstraZeneca is a non-replicating viral vector vaccine that stimulates an immune response against the coronavirus spike protein. One dose $(0.5 \mathrm{~mL})$ contains no less than $2.5 \times$ 108 infectious units of chimpanzee adenovirus encoding the SARS-CoV-2 spike glycoprotein (ChAdOX1S) [11]. The CoronaVac vaccine was given as a two-dose regimen administered 21 days apart and prioritized for adults aged 18-59 years. AZD1222 was given as a two-dose regimen administered 10 weeks apart for adults $\geq 18$ years and prioritized for the elderly above 60 years of age.

\subsection{Blood samples}

Venous blood samples $(5 \mathrm{ml})$ samples were collected at various time points after second dose vaccination in participants who received heterologous prime/booster inactivated COVID-19 vaccine and adenoviral-vectored vaccine. For participants who received homologous CoronaVac and AZD1222 vaccines, venous blood samples $(5 \mathrm{ml})$ were collected at 21-35 days after the second dose vaccination.

\subsection{Laboratory testing}

All sera samples were tested for SARS-CoV-2 spike RBD IgG by SARS-CoV-2 IgG II Quant assay (Abbott Diagnostics, Sligo, Ireland). This assay quantifies specific IgG against the RBD of the spike protein by using a chemiluminescent microparticle immunoassay (CMIA). The result was expressed as arbitrary units per milliliter $(\mathrm{AU} / \mathrm{mL}$ ) and the positive cut-off level was $\geq 50 \mathrm{AU} / \mathrm{mL}$ (upper limit: 40,000 AU/mL). Results of this assay had a good correlation with neutralizing antibodies [12].

The neutralizing activity in a subset of serum samples was tested against the original Wuhan strain and variants of concern, B.1.1.7, B.1.617.2 and B.1.351, using an enzyme-linked immunosorbent assay (ELISA)-based surrogate virus neutralization test (sVNT). For the original Wuhan strain SARS-CoV-2, sera samples were diluted and tested following the kit instructions for sVNT (Euroimmun, Lubeck, Germany). The positive cutoff was defined as $\geq 35 \%$ inhibition. The sVNT against wild type, B.1.1.7, B.1.351 and B.1.617.2 used cPassTM SARS-CoV-2 neutralizing antibody detection kit (GenScript, Jiangsu, China). The recombinant RBD of the SARS-CoV-2 spike protein contains N501Y for the B.1.1.7 variant, N501Y, E484K, and $\mathrm{K} 417 \mathrm{~N}$ for the B.1.351 variant, and L452R and T478K for the B.1.617.2 varinat. Briefly, the sera samples were diluted 1:10 with sample dilution buffer and then incubated with RBD-horseradish peroxidase (HRP) for $30 \mathrm{~min}$ at $37^{\circ} \mathrm{C}$ [13]. Then $100 \mu \mathrm{L}$ of the sample mixture was subsequently added to a capture plate with pre-coated h-angiotensin-converting enzyme 2 (ACE2) protein and incubated for 15 min at $37^{\circ} \mathrm{C}$. After a washing step, $100 \mu \mathrm{L}$ of 3,3',5,5'-tetramethylbenzidine (TMB) solution was added and 
the plate incubated in the dark for $15 \mathrm{~min}$ at $20-25^{\circ} \mathrm{C}$. Then $50 \mu \mathrm{L}$ of a stop solution was added to quench the reaction and the sample was read immediately at $450 \mathrm{~nm}$. The percent inhibition of a sample was calculated as (1-average optical density (OD) of sample/average OD of negative control) $\times 100 \%$. Greater than or equal to $30 \%$ inhibition was considered indicative of the presence of neutralizing antibodies.

\subsection{Statistical analysis}

Baseline characteristics are reported as means and standard deviations (SD). IgG S1/RBD antibodies titers are presented as geometric mean titer (GMT) and 95\% confidence interval (Cl). The sVNT antibodies titers are presented as median with interquartile range. The difference of spike RBD-specific IgG and percentage of inhibition between groups was calculated using the Mann-Whitney $U$ test. Statistical analysis was done using Prism 8.0 (GraphPad, San Diego, CA). A P-value $<0.05$ was considered statistically significant.

\section{Results}

\subsection{Demographic data}

All participants were Thai; however, the mean age of participants in the homologous AZD1222 vaccine cohort was significantly higher than that in the homologous CoronaVac $(P<0.001)$ because of vaccine prioritization during initial implementation (Table 1). Unlike the homologous vaccination cohorts, there were variations in intervals between the first and second dose vaccinations among the heterologous CoronaVac/AZD1222 vaccinees (median: 26 days, IQR: 21-32 days). We analyzed the immunogenicity data of the heterologous CoronaVac/AZD1222 vaccinees in two sets. The first set included all available data. The second set included heterologous CoronaVac/AZD1222 vaccinees who received vaccines 1435 days apart and had their blood collected between 14 and 35 days post-second dose vaccination (Supplementary Fig. 1 and Supplementary Table 1). Differences between all available data and data from participants with similar characteristics among all groups were not significant.

Table 1. Characteristics of the participants in the homologous and heterologous prime/booster of CoronaVac and AZD1222 vaccine. 


\begin{tabular}{|c|c|c|c|c|}
\hline Characteristics & $(\mathrm{N}=80)$ & $(\mathbf{N}=77)$ & $(\mathrm{N}=80)$ & P-value \\
\hline Sex No. (\%) & & & & $0.124^{\dagger}$ \\
\hline Male & $32(40 \%)$ & $20(26 \%)$ & $31(38.8 \%)$ & \\
\hline Female & $48(60 \%)$ & $57(74 \%)$ & $49(61.2 \%)$ & \\
\hline Age yrs, mean (SD) & $42(9.7)^{*}$ & $38.66(9.6)$ & $48.1(17.8)^{*}$ & $<0.001^{\S}$ \\
\hline Underlying disease No. (\%) & $19(23.8 \%)$ & $13(16.9 \%)$ & $48(60 \%)$ & $0.024^{\dagger}$ \\
\hline Diabetes mellitus & 4 & 1 & 15 & \\
\hline Hypertension & 8 & 8 & 20 & \\
\hline Heart disease & 1 & 2 & 3 & \\
\hline Other health conditions & 6 & 2 & 10 & \\
\hline $\begin{array}{l}\text { Interval between } 1^{\text {st }} \text { and } 2^{\text {nd }} \\
\text { dose median }(I Q R)\end{array}$ & 21 & $26(21-32)$ & 70 & \\
\hline $\begin{array}{l}\text { Interval between } 2^{\text {nd }} \text { dose } \\
\text { and collection date median } \\
\text { (IQR) }\end{array}$ & $28(27-28)$ & $31(29-35)$ & $29(26-31)$ & \\
\hline
\end{tabular}

$\dagger$ Number of positive test results was computed using Fisher's exact test. P-value less than 0.05 was considered statistically significance.

$\S$ Mean were tested using t t-test, asterisk defined as the significant counterpart.

\subsection{Receptor-binding domain (RBD)-specific IgG and sVNT against wild-type}

RBD-specific IgG was detected in $100 \%$ of participants in all groups after dose 2 , with the GMT (95\% Cl) of 1006 (836.8-1209), 1207 (989.4-1472), and 3962 (3327-4718) AU/ml among the homologous CoronaVac, homologous AZD1222, and heterologous CoronaVac/AZD1222 groups, respectively. CoronaVac followed by AZD1222 vaccine induced higher levels of spike receptor-binding domain-specific IgG than two-dose CoronaVac and AZD1222 vaccines $(P<0.001)$ (Fig. 1A). 
The sVNT was based on antibody-mediated blockage of ACE2-spike protein-protein interaction. The percent inhibition represented the ability of sera from vaccinated individuals to block the interaction between the ACE2 receptor protein and the SARS-CoV-2 RBD, which reflects the neutralizing activity. Our results showed that CoronaVac/AZD1222 vaccine recipients had higher neutralizing activities against the original Wuhan (wild-type) than the homologous CoronaVac and AZD1222 vaccine recipients (Fig. 1B).

\subsection{Neutralizing activities against SARS-CoV-2 variants}

Our results showed that CoronaVac/AZD1222 vaccine recipients had higher neutralizing activities against the wild-type and all variants of concern than the recipients of the two-dose CoronaVac $(P<0.0001)$ (Fig. 2A-D). In addition, the AZD1222 vaccine recipients also had higher neutralizing activities against the wild-type and all variants of concern than in the recipients of the CoronaVac $(P<0.01)$. Comparison of neutralizing activities between the CoronaVac/AZD1222 and the homologous AZD1222 vaccine recipients showed similar activities against the wild-type and B.1.1.7 strain but higher neutralizing activities were shown in the heterologous CoronaVac/AZD1222 group against B.1.351 and B.1.617.2 than the homologous AZD1222 group (Fig. 2C and 2D).

Our study showed that there was a reduction in neutralizing activities of sera against B.1.1.7, B.1.351 and B.1.167.2 compared to the wild-type strain in all groups (Fig. 3A-C). The most affected variant of concern was B.1.351.

\section{Discussion}

As of August 2021, the Department of Disease Control, Ministry of Public Health, Thailand, has implemented policies designating the first vaccine dose to be the CoronaVac vaccine and the second dose to be the AZD1222 vaccine, with more than 300,000 individuals getting vaccinated under this policy. The reasons are the shortage of AZD1222 and the decreased effectiveness of the two-dose CoronaVac vaccine against the variants of concern that are circulating in Thailand.

Our study enrolled recipients who had received heterologous prime/booster inactivated COVID-19 vaccine and adenoviral-vectored vaccine and sought antibody testing following vaccination, and they were compared with homologous vaccine recipients. Our immunogenicity data has shown that RBD-specific IgG was detected in $100 \%$ of heterologous CoronaVac/AZD1222 recipients after the second dose, with a higher GMT than those elicited by the two-dose CoronaVac and AZD1222 vaccines. In addition, the CoronaVac/AZD1222 vaccine recipients had higher amounts of neutralizing activities against the original Wuhan, B.1.617.2 and the B.1.351 strain than did the recipients of two-dose CoronaVac and AZD1222. Although the extent of the efficacy of the heterologous regimen has not been studied, the comparatively high level of immunogenicity compared to the homologous AZD1222 regimen supports its use as an alternative schedule, with the added benefit of a shorter waiting period between doses. 
Researchers are investigating to determine the immune correlates of protection to use as surrogate endpoints for vaccine efficacy. In a recent preprint, protection against SARS-CoV-2 challenge in vaccinated non-human primates strongly correlated with levels of anti-S antibody binding and neutralizing activity [14]. Because of its ability to elicit a high RBD-specific IgG and neutralizing activity following two-dose vaccination, a heterologous prime/booster regimen with CoronaVac/AZD1222 may provide more protection than the homologous CoronaVac regimen. Regarding the reactogenicity of the heterologous regimen, 1,100 individuals received the heterologous CoronaVac/AZD1222 as reported by Ministry of Public Health, Thailand, between March and July, 2021. However, this retrospective study has not noted any subsequent serious adverse events reported in the National Vaccine Adverse Event Reporting System [15].

Several SARS-CoV-2 variants such as B.1.351 and B.1.671.2 have demonstrated their ability to evade vaccine-induced immunity [16]. A recent study has shown that two doses of AZD1222 had an efficacy of $10.4 \%$ against the B.1.351 [17]. Our study also showed a reduction in neutralizing activities of sera against B.1.1.7, B.1.167.2 and B.1.351 compared to the original wild-type strain. Nevertheless, the neutralizing activity against the circulating delta variant B.1.617.2 among the heterologous vaccine recipients was higher than the homologous CoronaVac group. This result implied an additional benefit against variants of concern in the heterologous CoronaVac/AZD1222 regimen compared to the two-dose CoronaVac regimen.

The high immunogenicity profile of the heterologous prime/booster regimen in this study is congruent with conclusions of the Com-COV study, which also found an increase in anti-spike RBD-specific IgG and neutralizing titers compared to the heterologous regimen ${ }^{1}$. In the Com-COV study, the researchers investigated combinations of the Pfizer mRNA vaccine (BNT162b2) and the AZD1222 vaccines available in the United Kingdom. Two-dose AZD1222 administered 6 weeks apart elicited a lower immune response than when given 10-12 weeks apart. The underlying mechanism is likely due to the host anti-adenoviral antibodies elicited by the first vaccination preventing the virus in the second vaccine dose to enter the cells when the second dose is given sooner than 10-12 weeks. Nevertheless, the Pfizer mRNA vaccine (BNT162b2) given as a second dose in AZD1222-primed individuals has been shown to induce a higher response than that of the AZD1222 given as a second dose. Heterologous vaccination regimens have been previously examined with experimental vaccines for HIV [18], malaria [19], and Ebola [20], a precedent for this regimen's efficacy; however, the mechanism for increased immunogenicity from mixing CoronaVac/AZD1222 has yet to be elucidated.

There are a few noteworthy limitations to the current study. Because our study participants were recruited in a real-world setting before the Ministry of Public Health launched the vaccine recommendation, the schedule of heterologous vaccination did not follow the recently released guideline stating that two doses should be given 28 days apart. In this study, approximately $80.5 \%$ of individuals received the first and second dose of the heterologous regimen at an interval between 14 and 35 days. Secondly, the timing of collecting blood samples after the second dose in the heterologous vaccine group was not the same as for the homologous vaccine group. This caveat has not statistically affected the conclusion but 
nonetheless should be considered. Third, the age demographic disparity was present between different vaccination regimen groups. The inherent nature of Thailand's vaccination policy, which prioritizes vaccination with the AZD1222 vaccine in elderly people, consequently led to a higher average age for the homologous AZD1222 regimen cohort. The increased average age in the homologous AZD1222 regimen cohort can lead to a lower immune response as also demonstrated in a study of immunogenicity of an RNA vaccine [21]. The age-related lower immune response is likely due to the "immunosenescence" phenomenon as a result of increases in terminally differentiated memory cell populations, lymph node fibrosis, and altered cytokine production among the elderly [22]. Lastly, cell-mediated immunity was not explored in this study.

In conclusion, a heterologous prime/booster regimen demonstrated a strong immune response. Further studies are underway to determine the reactogenicity and immunogenicity of a heterologous prime/booster regimen in clinical trials against other SARS-CoV-2 variants. With an acceptable immunogenicity profile, here is the first report to show that a heterologous prime/booster regimen with CoronaVac/AZD1222 would provide greater flexibility for countries experiencing supply difficulties and individuals with adverse events following the first dose CoronaVac vaccination.

\section{Declarations}

\section{Data Availability}

All data generated during this study are contained within this manuscript and its Supplementary Information files.

\section{Acknowledgements}

This work was supported by The National Research Council of Thailand (NRCT), Health Systems Research Institute, The Center of Excellence in Clinical Virology of Chulalongkorn University, and King Chulalongkorn Memorial Hospital. Nungruthai Suntronwong was supported by the second Century Fund (C2F) fellowship of Chulalongkorn University.

\section{Conflict of Interest}

The authors declare no conflict of interest.

\section{References}

[1] Liu X, Shaw RH, Stuart ASV, Greenland M, Aley PK, Andrews NJ, et al. Safety and immunogenicity of heterologous versus homologous prime-boost schedules with an adenoviral vectored and mRNA COVID-19 vaccine (Com-COV): a single-blind, randomised, non-inferiority trial. Lancet 2021; S01406736(21): 01694-9. 
[2] Borobia AM, Carcas AJ, Pérez-Olmeda M, Castaño L, Bertran MJ, García-Pérez J, et al. CombiVacS Study Group. Immunogenicity and reactogenicity of BNT162b2 booster in ChAdOx1-S-primed participants (CombiVacS): a multicentre, open-label, randomised, controlled, phase 2 trial. Lancet 2021; 398(10295):121-130.

[3] Barros-Martins J, Hammerschmidt SI, Cossmann A, Odak I, Stankov MV, Morillas Ramos G, et al. Immune responses against SARS-CoV-2 variants after heterologous and homologous ChAdOx $1 \mathrm{nCoV}$ 19/BNT162b2 vaccination. Nat Med 2021; Jul 14.

[4] Schmidt T, Klemis V, Schub D, Mihm J, Hielscher F, Marx S, et al. Immunogenicity and reactogenicity of a heterologous COVID-19 prime-boost vaccination compared with homologous vaccine regimens. medRxiv 2021; 2021.06.13.21258859.

[5] Palacios R, Patiño EG, de Oliveira Piorelli R, Conde MTRP, Batista AP, Zeng G, et al. Double-Blind, Randomized, Placebo-Controlled Phase III Clinical Trial to Evaluate the Efficacy and Safety of treating Healthcare Professionals with the Adsorbed COVID-19 (Inactivated) Vaccine Manufactured by Sinovac PROFISCOV: A structured summary of a study protocol for a randomised controlled trial. Trials 2020; 21(1):853.

[6] Tanriover MD, Doğanay HL, Akova M, Güner HR, Azap A, Akhan S, et al. Efficacy and safety of an inactivated whole-virion SARS-CoV-2 vaccine (CoronaVac): interim results of a double-blind, randomised, placebo-controlled, phase 3 trial in Turkey. Lancet 2021; 398(10296):213-222.

[7] Bueno SM, Abarca K, González PA, Gálvez NM, Soto JA, Duarte LF et al. Interim report: Safety and immunogenicity of an inactivated vaccine against SARS-CoV-2 in healthy chilean adults in a phase 3 clinical trial. medRxiv 2021; 2021.03.31.212544948.

[8] Voysey M, Costa Clemens SA, Madhi SA, Weckx LY, Folegatti PM, Aley PK, et al. Single-dose administration and the influence of the timing of the booster dose on immunogenicity and efficacy of ChAdOx1 nCoV-19 (AZD1222) vaccine: a pooled analysis of four randomised trials. Lancet 2021; 397(10277):881-891.

[9] Tanakasempipat $P$, Thepgumpanat $P$. Thailand sticks with Sinovac vaccine after cases of 'strokelike' side effects. Reuters (2021) [Accessed 06/23/2021] ; Available from:

https://www.reuters.com/world/asia-pacific/thailand-sticks-with-sinovac-vaccine-after-cases-stroke-likeside-effects-2021-04-21/.

[10] Corbett KS, Nason MC, Flach B, Gagne M, O' Connell S, Johnston TS, et al. Immune Correlates of Protection by mRNA-1273 Immunization against SARS-CoV-2 Infection in Nonhuman Primates. bioRxiv [Preprint] 2021; Apr 23:2021.04.20.440647.

[11] Division of Epidemiology, Department of Disease Control, Ministry of Public Health. Situation summary of heterologous immunization with Sinovac's and AstraZeneca's COVID-19 vaccines in 
Thailand (2021) [Accessed 06/23/2021]; Available from: https://wesr.doe.moph.go.th/wesr_new/file/y64/H64282021071120210717.pdf.

[12] Krause PR, Fleming TR, Longini IM, Peto R, Briand S, Heymann DL, et al. SARS-CoV-2 Variants and Vaccines. N Engl J Med 2021; 385(2):179-186.

[13] Madhi SA, Baillie V, Cutland CL, Voysey M, Koen AL, Fairlie L, et al. Efficacy of the ChAdOx1 nCoV19 Covid-19 Vaccine against the B.1.351 Variant. N Engl J Med 2021; 384(20):1885-1898.

[14] Rerks-Ngarm S, Pitisuttithum P, Nitayaphan S, Kaewkungwal J, Chiu J, Paris R, et al. Vaccination with ALVAC and AIDSVAX to prevent HIV-1 infection in Thailand. N Engl J Med 2009; 361(23):2209-20.

[15] McConkey SJ, Reece WH, Moorthy VS, Webster D, Dunachie S, Butcher G, et al. Enhanced T-cell immunogenicity of plasmid DNA vaccines boosted by recombinant modified vaccinia virus Ankara in humans. Nat Med 2003; 9(6):729-35.

[16] Pollard AJ, Launay O, Lelievre JD, Lacabaratz C, Grande S, Goldstein N, et al. Safety and immunogenicity of a two-dose heterologous Ad26.ZEBOV and MVA-BN-Filo Ebola vaccine regimen in adults in Europe (EBOVAC2): a randomised, observer-blind, participant-blind, placebo-controlled, phase 2 trial. Lancet Infect Dis 2021; 21(4):493-506.

[17] Maeda K, Amano M, Uemura Y, Tsuchiya K, Matsushima T, Noda K, et al. Correlates of Neutralizing/SARS-CoV-2-S1-binding Antibody Response with Adverse Effects and Immune Kinetics in BNT162b2-Vaccinated Individuals. medRxiv [Preprint] 2021; Jul 30:2021.07.27.21261237.

[18] Crooke SN, Ovsyannikova IG, Poland GA, Kennedy RB. Immunosenescence and human vaccine immune responses. Immun Ageing 2019; 16:25.

[19] Thailand Food and Drug Administration. Summary of Product Characteristic CoronaVac (2021) [Accessed 06/23/2021]; Available from: https://www.fda.moph.go.th/sites/drug/Shared\%20Documents/Vaccine/U1DR1C1072640000311C-SPCEN.pdf .

[20] European Medicines Agency. COVID-19 Vaccine AstraZeneca (2021) [Accessed 06/23/2021]; Available from: https://www.ema.europa.eu/en/documents/product-information/covid-19-vaccineastrazeneca-product-information-approved-chmp-29-january-2021-pending-endorsement_en.pdf .

[21] Perkmann T, Perkmann-Nagele N, Koller T, Mucher P, Radakovics A, Marculescu R, et al. Anti-Spike Protein Assays to Determine SARS-CoV-2 Antibody Levels: a Head-to-Head Comparison of Five Quantitative Assays. Microbiol Spectr 2021; e0024721.

[22] Tan CW, Chia WN, Qin X, Liu P, Chen MI, Tiu C, et al. A SARS-CoV-2 surrogate virus neutralization test based on antibody-mediated blockage of ACE2-spike protein-protein interaction. Nat Biotechnol 2020; 38(9):1073-1078. 

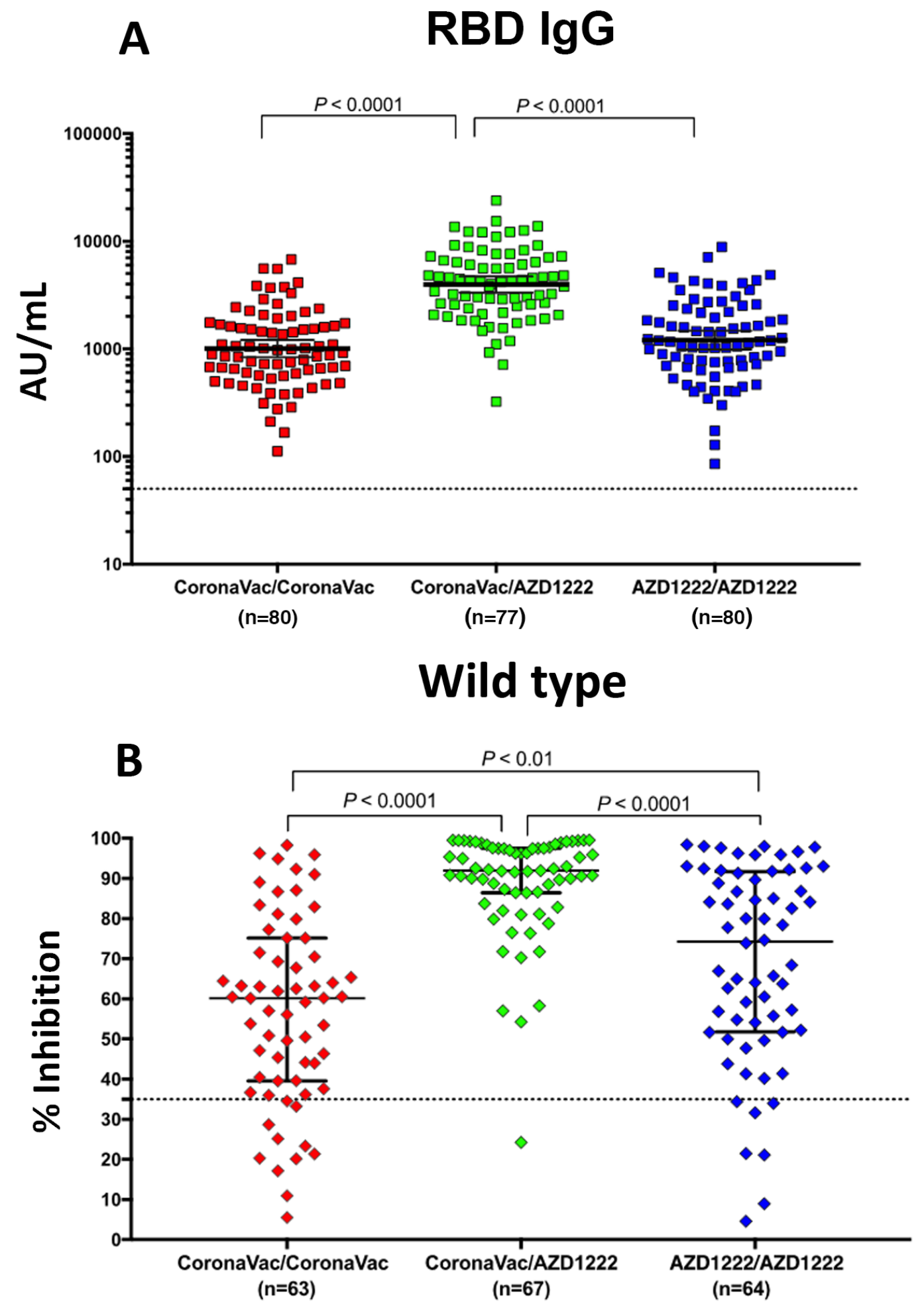

Figure 1

RBD-specific IgG antibodies and neutralizing activities against SARS-CoV-2 original Wuhan strain following heterologous CoronaVac/AZD1222 and homologous CoronaVac or AZD1222 vaccination. (A) RBD-specific IgG titers obtained at 28 days after homologous CoronaVac/CoronaVac (red), 14-72 days 
after CoronaVac/AZD1222 (green), and 28 days after homologous AZD1222/AZD1222 (blue). (B) Percentage of inhibition against Wuhan strain using a surrogate virus neutralization test (sVNT) (Euroimmun, Lubeck, Germany). Bars represent GMT (95\% Cl) for RBD-specific IgG and median (IQR) for sVNT.

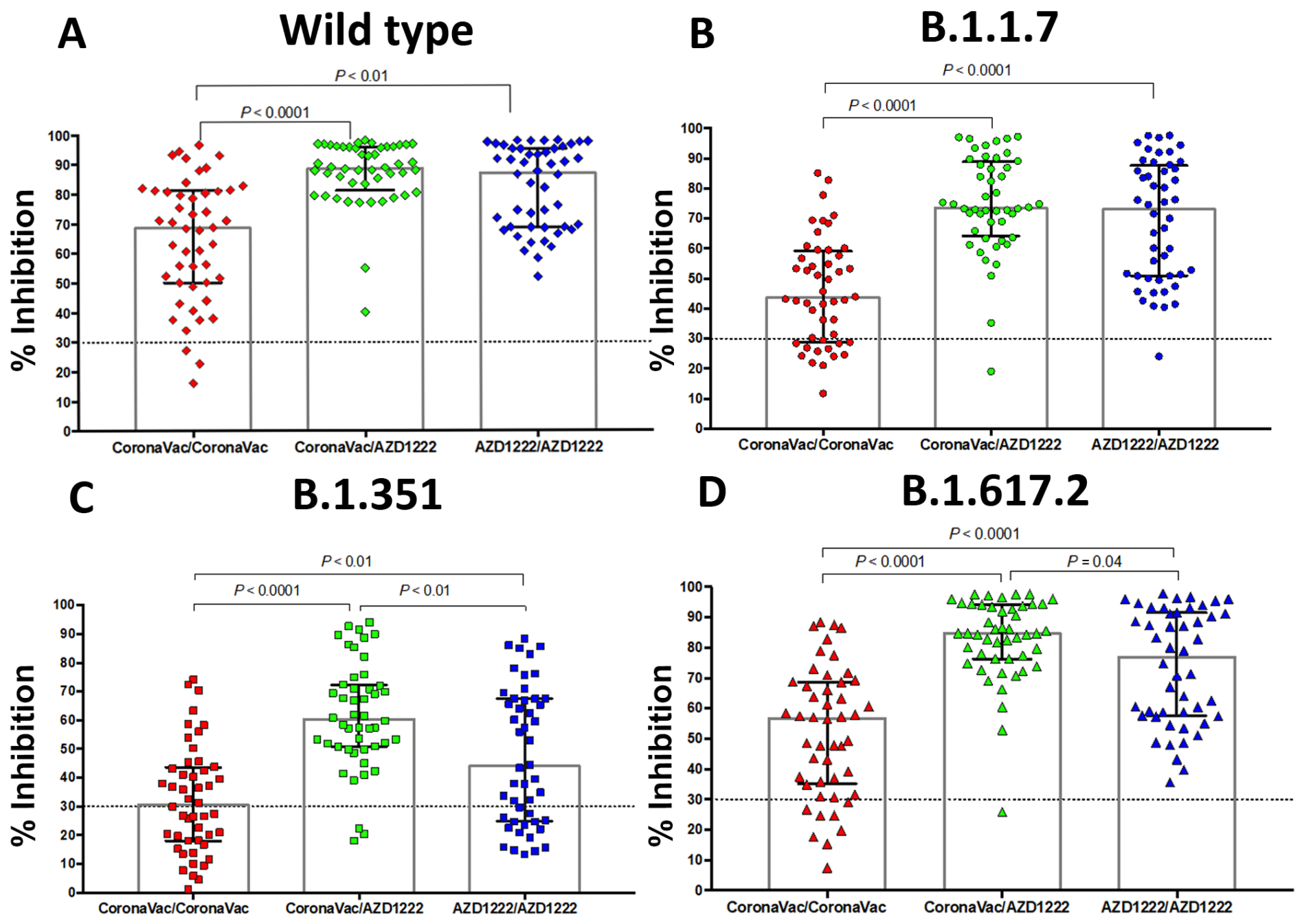

Figure 2

Neutralizing activities against SARS-CoV-2 wild-type and variants following heterologous CoronaVac/AZD1222 and homologous CoronaVac or AZD1222 vaccination. Percentage of inhibition against (A) Wild type (rhombus), (B) B.1.1.7 (circle), (C) B.1.351 (square) and (D) B.1.617.2 (triangle) SARS-CoV-2 measured using a surrogate virus neutralization test (sVNT); cPassTM SARS-CoV-2 neutralizing antibody detection kit (GenScript, Jiangsu, China). Bars represent median (IQR). 

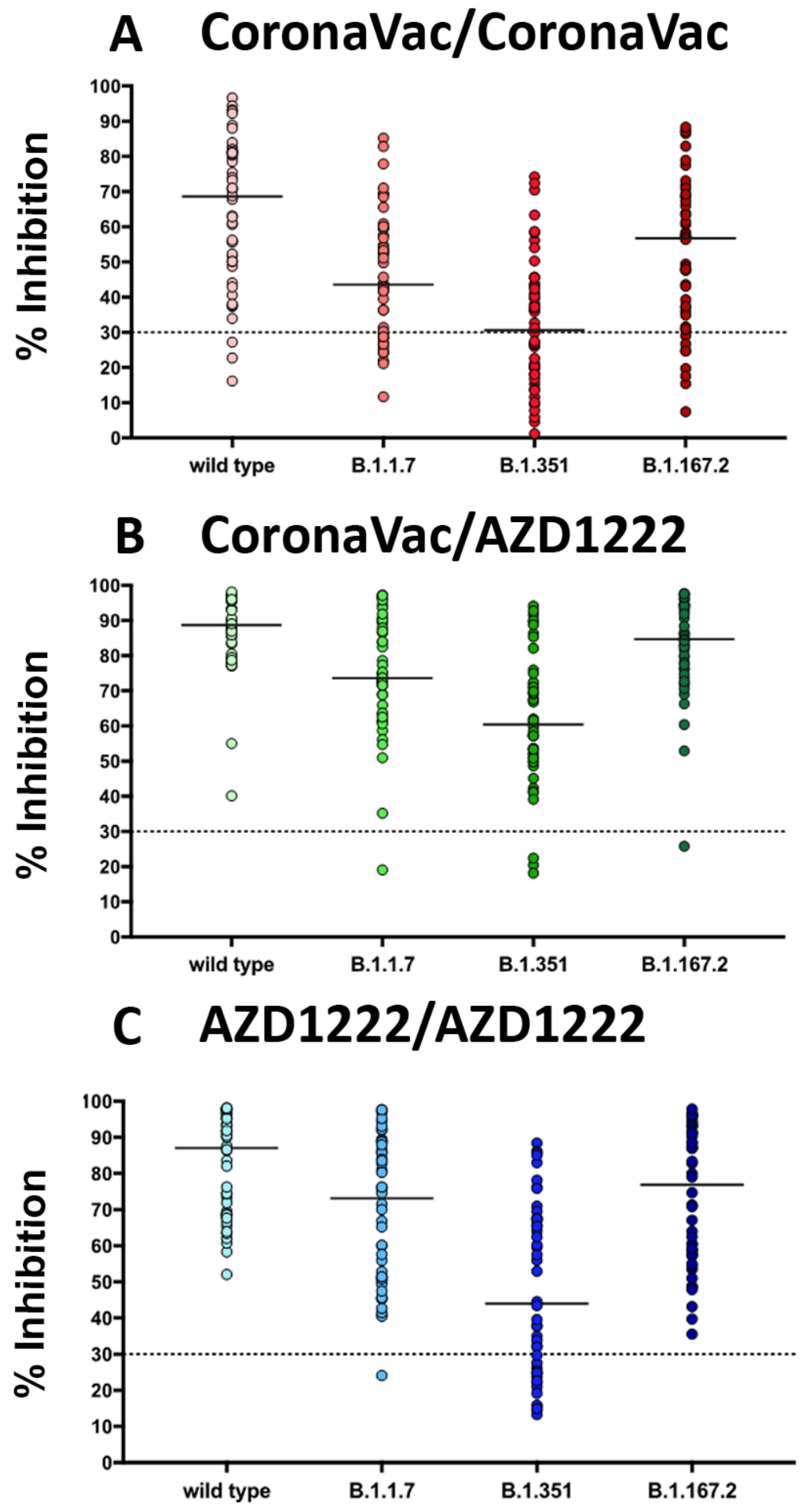

Figure 3

Comparison between neutralizing activities against variants relative to the wild type in serum samples obtained from (A) homologous CoronaVac/CoronaVac, (B) heterologous CoronaVac/AZD1222, and (C) homologous AZD1222/AZD1222 vaccinees. The lines indicate the median of \% inhibition.

\section{Supplementary Files}


This is a list of supplementary files associated with this preprint. Click to download.

- S1Fig.tif

- SupplementaryTable120210828.docx 